Purpose To report the introduction of a gravimetric process of weighing to encourage its future implementation and increase the quality and safety in the preparation of parenteral nutrition (PN). Materials and Methods In order to standardise the gravimetric control of PN, a protocol was developed by the nutrition unit. The quality of the PN preparation was established by calculating the accuracy (the mean of the error in the gravimetric analyses (EGA)) and precision (square root of the mean square of the EGA) and the alert limits were set at $\pm 5 \%$.

The first step was to determinate the densities of the components of the PN and update the parenteral nutrition programme. The PN labels were modified to show the theoretical weight of the $\mathrm{PN}$ and the maximum and minimum limits allowed.

Results One strategy established for the quality control of the final product was to compare the final weight of the product with the volume and the density calculated for each component.

In the first 67 days 150 parenteral nutrition mixtures were made in the neonatology department. The average theoretical weight was $323.68 \mathrm{~g}( \pm 236.04)$ and the average measured weight was $323.45 \mathrm{~g}$ $( \pm 239.94)$

The mean difference of the actual weight versus the theoretical was $2.8 \%( \pm 0.04)$.

Conclusions Gravimetric analysis is a strategy to cheque the accuracy and precision in $\mathrm{PN}$ and complements the quality assurance processes normally used to regulate the preparation.

No conflict of interest.

\section{GRP-091 IMPLEMENTATION OF KEY PERFORMANCE INDICATORS IN CYTOTOXIC COMPOUNDING UNITS}

doi:10.1136/ejhpharm-2013-000276.091

TK Wrønding, C Øby. Region Hovedstadens Apotek, KFS Serviceproduktionen Copenhagen, Denmark

Background The Capital Region Hospital pharmacy prepares more than 90,000 bags of cytotoxic treatments a year. There has been no tradition in the pharmacy of systematically monitoring essential parameters in the productions units. Because of an increasing need for treatments on the wards, the delivery time for cytotoxics went up to 5 hours, resulting in complaints from patients. The number of products that were rejected was very high. In 2010, the cost of rejected products was more than $€ 200,000$. Analysing and addressing root causes when nonconformities arose could take up to one year.

Purpose To reduce production time and make it more stable while improving quality and reducing costs.

Materials and Methods Three Key Performance Indicators (KPIs) were introduced: Delivery, Rejects and GMP non-conformities, in order to ensure a stable, short production time and a constant focus on cost and product quality. The three KPIs are continuously monitored and posted on boards in the production area. The KPIs are discussed with the staff in weekly meetings.

Results Overall delivery time has been reduced. $75 \%$ of patients are now waiting less than $1 \frac{1}{2}$ hours and $90 \%$ of the pre-ordered treatments are delivered on time.

1. The number and types of rejects are now known. The goal is to reduce the value of rejects by $15 \%$ in 2012 .

2. Processing time for non-conformities is now a maximum of 21 days.

Conclusions By defining the relevant KPIs, and having an ongoing dialogue with employees about the KPIs, it has been possible to significantly increase awareness among the employees of the overall performance of the production process. The increased awareness has resulted in a significantly improved performance that provides value for our patients.

No conflict of interest.

\section{GRP-092 IMPLEMENTATION OF RECOMMENDATIONS ARISING FROM THERAPEUTIC MONITORING OF VANCOMYCIN TROUGH LEVELS IN A TERTIARY HOSPITAL}

doi:10.1136/ejhpharm-2013-000276.092

M Dominguez Cantero, C Gallego Muñoz, ME Rodriguez Mateos, MV Manzano Martin, L Obel Gil, R Bulo Concellon, M Ladrón De Guevara García, MJ Huertas Fernandez, I Moyano Prieto, JM Rodriguez Camacho. H.U. Puerta del Mar, Servicio de Farmacia, Cádiz, Spain

Background In 2009 we established a consensus review of therapeutic monitoring of vancomycin by several societies including the American Society of Health-System Pharmacists (ASHP), the Infectious Diseases Society of America (IDSA) and the Society of Infectious Diseases Pharmacists (SIDP).

Purpose To study the use of plasma concentrations (PCs) as a tool for monitoring the effectiveness and safety of vancomycin treatment; follow-up of the changes made in response to the recommendations made.

Materials and Methods Retrospective descriptive study in a tertiary hospital during the first four months of 2012. Vancomycin trough plasma concentrations (PCs) were collected. The laboratory service requested and identified patients treated with vancomycin in the unit dose dispensing system. An automatic system recorded the doses, days of treatment indicated, glomerular filtration rate (GF) prior to and during treatment with vancomycin (calculated by the MDRD formula, considering renal function impairment (RFI) lower GFR $80 \mathrm{ml} / \mathrm{min}$ ) and concomitant treatment. The recommendations contained in the consensus document on vancomycin therapeutic monitoring of the Infectious Diseases Society of America (IDSA) and the American Society of Health-System Pharmacists (ASHP) were used as the standard criteria for vancomycin PC monitoring: RFI, treatment for more than five days or concomitant nephrotoxic drug administered.

Results 30 patients were enrolled, median age 66, 21 men, median treatment duration of 7 (1-46) days. The PC was checked in $10 \%$ (3) of the patients, two had PCs within the recommended values. In the third patient a single plasma level was requested, which revealed less than the recommended values but the same dose and schedule was maintained throughout the 29 days of treatment. In none was the area under the curve divided by the minimum inhibitory concentration (AUC/MIC) reported, the pharmacokinetic parameter best related to the effectiveness of vancomycin. Plasma levels were not requested in 27 patients, $90 \%$ of the total treated with vancomycin. $63 \%$ met one or more criteria for monitoring; treatment for longer than five days was the most common criterion (20). In one patient the recommendations made were acted on.

Conclusions The established recommendations on therapeutic monitoring of vancomycin are not being applied in our hospital.

A high number of patients treated with vancomycin did not use the PC as a parameter with which to monitor the efficacy and safety of antibiotic treatment.

One possible cause could be a lack of training of medical staff on the usefulness and benefits of vancomycin monitoring, particularly during prolonged treatment and in patients with RFI.

No conflict of interest.

\section{GRP-093 IMPLEMENTING AND IMPROVING MEDICINES RECONCILIATION ON ADMISSION AT NORTH BRISTOL NHS TRUST (NBT)}

doi:10.1136/ejhpharm-2013-000276.093

'1 Smith, ' $\mathrm{J}$ Hamer, ${ }^{1} \mathrm{~A}$ Mundell, 'N Mogford, ${ }^{1} \mathrm{R}$ Brown, ${ }^{2} \mathrm{~F}$ Hamill. ${ }^{1}$ North Bristol NHS Trust, Pharmacy, Bristol, UK; ${ }^{2}$ North Bristol NHS Trust, Clinical Audit \& Assurance, Bristol, UK

Background Medicines Reconciliation ensures that medicines prescribed on patient admission correspond to those taken before 
admission. This process involves discussion with patients/carers/ using primary care records.

Medicines errors cause harm to patients, lead to increased morbidity/mortality/inflated healthcare costs [1,2]

NBT has invested in many safety initiatives including: the Safer Patients Initiative (SPI2) and the Southwest Quality and Patient Safety Improvement Programme (SWOPSI).

Purpose To implement and improve Medicines Reconciliation. The objectives were to: Ensure more than $95 \%$ of patients admitted receive Medicines Reconciliation within 24 hours; Improve the quality of Medicines Reconciliation and reduce medicines errors on admission.

Materials and Methods Using improvement methodology, tests of change were trialled and spread, involving:

- Phase 1: 2007-2008: (1-8 wards)

- Introduced a Medicines Admissions Proforma

- Developed an e-audit tool

- Phase 2: 2008-2009: (8-11 wards)

- Training DVD was designed

- Analysed admissions data to spread towards where admissions were $>2 \%$ of the total number of admissions

- Collected randomised data electronically as a run chart

- Improved communication (Patients/Ambulance/GP's)

- Phase 3: 2009-now: (11-30 wards)

- 2010: tests of change on accuracy of Medicines Reconciliation, spreading to 42 wards

- 2012: Surgical Pharmacist funding agreed following a Pre-admissions clinic trial.

Results The medians in the table show improvements 2007-2012. In 2011 we achieved and maintained our target. Accuracy data showed only $55 \%$ of admissions drug histories taken by doctors alone are accurate.

Conclusions From February 2011 we achieved and maintained our $95 \%$ target on 30 wards. We improved the quality of medicines reconciliation and reduced medicines errors on admission.

The Institute for Healthcare Improvement congratulated us and OIPP's national programme benchmarking teaching hospitals also highlights our remarkable results.

\begin{tabular}{lc} 
Abstract GRP-093 Table 1 \\
\hline Date & Median\% \\
\hline May 2007 & $60 \%$ \\
July-Dec 2007 & $56 \%$ \\
Jan-Jun 2008 & $67 \%$ \\
Jul-Dec 2008 & $73 \%$ \\
Jan-Jun 2009 & $77 \%$ \\
Jul-Dec 2009 & $77 \%$ \\
Jan-Jun 2010 & $85 \%$ \\
Jul-Dec 2010 & $92 \%$ \\
Jan-Jun 2011 & $96 \%$ \\
Jul-Dec 2011 & $95 \%$ \\
Jan-Jun 2012 & $95 \%$ \\
Sep 2012 & $98 \%$ \\
\hline
\end{tabular}

\section{References}

1. National Institute for Health and Clinical Excellence/National Patient Safety Agency: Medicines Reconciliation guidance

2. Quality, Innovation, Productivity and Prevention (OIPP) including Medicines Optimisation and Transfer of Care

No conflict of interest.

\section{GRP-094 IMPROVEMENT OF THE CLINICALLY RELEVANT SAFETY OF CHEMOTHERAPY BY THE INVOLVEMENT OF A CLINICAL PHARMACIST}

doi:10.1136/ejhpharm-2013-000276.094

${ }^{1} \mathrm{~N}$ Hohn, ${ }^{1} \mathrm{~S}$ von Hobe, ${ }^{2} \mathrm{~T}$ Brümmendorf, ${ }^{2} \mathrm{O}$ Galm, ${ }^{2} \mathrm{E}$ Jost, ${ }^{\prime} \mathrm{A}$ Eisert. ${ }^{1}$ University Hospital of the RWTH Aachen, Pharmacy, Aachen, Germany; ${ }^{2}$ University Hospital of the RWTH Aachen, Oncology, Aachen, Germany
Background To avoid medical errors and thus to improve the safety and quality of cancer treatment in our institution, all chemotherapy prescriptions are critically checked by a clinical pharmacist. Prescription errors are communicated immediately to the attending physician and corrected prior to the preparation and administration of the drugs.

Purpose To compile error statistics and to assess the potential severity of errors in chemotherapy prescriptions, we retrospectively analysed and evaluated prescription errors in order to improve the safety of treatment.

Materials and Methods 42624 paper written (no CPOE) chemotherapy prescriptions (containing 86101 prescriptions for medicines) from 19 departments of the University Hospital of RWTH Aachen between 2004 and 2009 were analysed retrospectively by the hospital pharmacy. The most important criteria for analysis were wrong patient, wrong drug, missing drug, wrong dose, wrong application day and wrong protocol. The clinical relevance of the medical errors detected was assessed independently by four oncologists and two clinical pharmacists using the criteria of Small et al, [1].

Results In total, 696 medicines errors were detected in 373 prescriptions during the routine verification by the pharmacist. By far the most abundant errors $(92.4 \%$ of the total) were related to the dose. Of the 373 prescriptions the team reviewed $20 \%$ of the errors as minor, $50 \%$ as significant, $25 \%$ as severe and $5 \%$ as potentially fatal. Potentially fatal errors were detected in regard to overdoses and once to the prescription of the wrong drug.

Conclusions Our results clearly show the relevance of clinical pharmacists being part of the therapeutic team to reduce medicines errors and to prevent any patient harm.

\section{Reference}

1. Small MD, Barrett A, Price GM. The impact of computerized prescribing on error rate in a department of Oncology/Hematology. J Oncol Pharm Pract 2008;14:181-7.

No conflict of interest.

\section{GRP-095 IMPROVING MEDICATION SAFETY: THE DANAPAROID STOREY}

doi:10.1136/ejhpharm-2013-000276.095

${ }^{1} \mathrm{M}$ Trojan, ${ }^{2} \mathrm{~A}$ Ihbe-Heffinger, ${ }^{3} \mathrm{~A}$ Greinacher, ${ }^{4} \mathrm{C}$ Unkrig, ${ }^{1} \mathrm{~A}$ Müller, ${ }^{1} \mathrm{R}$ Bernard ${ }^{1} \mathrm{C}$ Querbach. ${ }^{1}$ Klinikum Rechts der Isar der TUM München, Pharmacy, München, Germany; ${ }^{2}$ Klinikum Rechts der Isar der TUM München, Department of Gynecology, München, Germany; ${ }^{3}$ Department of Immunology and Transfusion Medicine, Medicine, Greifswald, Germany; ${ }^{4}$ Federal Institute for Drugs and Medical Devices (BfArM), Drug Safety, Bonn, Germany

Background During rounds a clinical pharmacist identified and corrected subtherapeutic doses of danaparoid. This error was caused by misleading information in the German Summary of Product Characteristics (SPC)

Purpose To improve medication safety an in-house standard operation procedure for the use of danaparoid sodium was implemented and changes in the SPC were requested.

Materials and Methods The error frequency when using danaparoid was determined over a period of 4 months. The medicines information centre intensified the routine cheque of prescriptions for danaparoid as well as the counselling on dose adjustment. Medication errors were reported to the manufacturer and the Federal Institute for Drugs and Medical Devices (BfArM). At the same time an interdisciplinary working group developed in-house dosing recommendations. Suggestions for modifications of the SPC were submitted to the BfArM

Results From April to July 2011 subtherapeutic doses were detected in 7 of 21 patients treated with danaparoid at the university hospital Klinikum rechts der Isar: because of misleading information in the SPC, prophylactic doses were administered despite indications for therapeutic anticoagulation. In July 2011 the results 